\title{
Optimized Schwarz Methods for Heterogeneous Helmholtz and Maxwell's Equations
}

\author{
Victorita Dolean ${ }^{1}$, Martin J. Gander ${ }^{2}$, Erwin Veneros ${ }^{3}$, Hui Zhang ${ }^{4}$
}

\section{Introduction}

The Helmholtz equation is very difficult to solve by iterative methods [18], and the time harmonic Maxwell's equations inherit these difficulties. Optimized Schwarz methods are among the most promising iterative techniques. For the Helmholtz equation, they have their roots in the seminal work of Deprés $[6,7]$, which led to the development of optimized transmission conditions $[5,19,21,20,3]$, and these techniques were independently rediscovered for the sweeping preconditioner [17] and the source transfer domain decomposition method [4]. For the time harmonic Maxwell's equations, optimized transmission conditions were developed and tested for problems without conductivity in $[1,11,23,24,16]$, and with conductivity in [8]. Particular Galerkin discretizations of transmission conditions were studied in [13, 12], and for scattering applications, see [23, 24].

In $[14,15]$, it was discovered that heterogeneous media can actually improve the convergence of optimized Schwarz methods, provided that the coefficient jumps are aligned with the interfaces, and the jumps are taken into account in an appropriate way in the transmission conditions. Similar results were found for Maxwell's equations in [9] and [10]; it is even possible to obtain convergence independently of the mesh size in certain situations. We present and study here transmission conditions for the Helmholtz equation with heterogeneous media, and establish a relation to the results of $[9,10]$ written for Maxwell's equations. We then study improved convergence behavior for specific choices of the discretization parameters related to the pollution effect $[2]$.

Section de mathématiques, Université de Genève, 1211 Genève 4 victorita.dolean@unige.ch · martin.gander@unige.ch · erwin.veneros@unige.ch . Key Laboratory of Oceanographic Big Data Mining \& Application of Zhejiang Province, supported by Research Start Funding of Zhejiang Ocean University, Zhoushan 316022, China huiz@zjou.edu.cn 


\section{Optimized Schwarz Methods for Helmholtz and Maxwell's Equations}

We consider the two dimensional Helmholtz equation in discontinuous media with piece-wise constant density $\rho$ and wave-speed $c$. The Helmholtz equation in $\Omega=\mathbb{R}^{2}$ is defined by

$$
\nabla\left(\frac{1}{\rho} \nabla \cdot u\right)+\frac{\omega^{2}}{c^{2} \rho} u=f, \text { in } \Omega,
$$

with

$$
\rho=:\left\{\begin{array}{ll}
\rho_{1} & \text { in } \Omega_{1}, \\
\rho_{2} & \text { in } \Omega_{2},
\end{array} \quad c:= \begin{cases}c_{1} & \text { in } \Omega_{1}, \\
c_{2} & \text { in } \Omega_{2},\end{cases}\right.
$$

where $\Omega_{1}=\mathbb{R}^{-} \times \mathbb{R}, \Omega_{2}=\mathbb{R}^{+} \times \mathbb{R}$ and the Sommerfeld radiation condition is imposed at infinity,

$$
\lim _{|x| \rightarrow \infty} \sqrt{|x|}\left(\partial_{|x|} u+i \omega u\right)=0
$$

for every possible direction $\frac{x}{|x|}$.

We can naturally define a Schwarz algorithm for equation (1) with Robin transmission conditions at the interface aligned with the discontinuity between the coefficients, and parameters $s_{1}, s_{2} \in \mathbb{C}$,

$$
\begin{aligned}
\nabla\left(\frac{1}{\rho_{1}} \nabla \cdot u_{1}^{n}\right)+\frac{\omega^{2}}{c_{1}^{2} \rho_{1}} u_{1}^{n} & =f, & & \text { in } \Omega_{1}, \\
\left(\frac{1}{\rho_{1}} \partial_{n_{1}}+\frac{1}{\rho_{2}} s_{2}\right) u_{1}^{n} & =\left(\frac{1}{\rho_{2}} \partial_{n_{1}}+\frac{1}{\rho_{2}} s_{2}\right) u_{2}^{n-1}, & & \text { on } \Gamma, \\
\nabla\left(\frac{1}{\rho_{2}} \nabla \cdot u_{2}^{n}\right)+\frac{\omega^{2}}{c_{2}^{2} \rho_{1}} u_{2}^{n} & =f, & & \text { in } \Omega_{2}, \\
\left(\frac{1}{\rho_{2}} \partial_{n_{2}}+\frac{1}{\rho_{1}} s_{1}\right) u_{2}^{n} & =\left(\frac{1}{\rho_{1}} \partial_{n_{2}}+\frac{1}{\rho_{1}} s_{1}\right) u_{1}^{n-1}, & & \text { on } \Gamma .
\end{aligned}
$$

Proposition 1. The convergence factor of algorithm (3) is given by

$$
\rho_{\text {opt }}\left(k, \rho_{1}, \rho_{2}, \omega, c_{1}, c_{2}, s_{1}, s_{2}\right)=\left|\frac{\left(\lambda_{1}-s_{1}\right)\left(\lambda_{2}-s_{2}\right)}{\left(\lambda_{1}+s_{2} \frac{\rho_{1}}{\rho_{2}}\right)\left(\lambda_{2}+s_{1} \frac{\rho_{2}}{\rho_{1}}\right)}\right|^{1 / 2},
$$

with $\lambda_{j}=\sqrt{k^{2}-\omega_{j}^{2}}, \omega_{j}=\frac{\omega}{c_{j}}$ for $j=1,2$.

The proof of Proposition 1 is based in Fourier analysis, see [27] for details.

In order to obtain an efficient algorithm, we have to choose $s_{1}$ and $s_{2}$ such that $\rho_{\text {opt }}$ becomes as small as possible for all relevant numerical frequencies $k \in K:=\left[k_{\min }, k_{\max }\right]$, where $k_{\min }$ is the lowest relevant frequency $\left(k_{\min }\right.$ depends on the geometry of the media) and $k_{\max }=\frac{c_{\max }}{h}$ is the highest numerical frequency supported by the numerical grid with mesh size $h$.

In what follows, we only consider $s_{1}=P_{1}(1+i)$ and $s_{2}=P_{2}(1+i), P_{j}>0$, a choice that has been justified in [21], and thus study the min-max problem 


$$
\rho_{\mathrm{opt}}^{*}=\min _{P_{1}, P_{2}>0} \max _{k \in K}\left|\rho_{\mathrm{opt}}\left(k, \rho_{1}, \rho_{2}, \omega, c_{1}, c_{2}, P_{1}(1+i), P_{2}(1+i)\right)\right| .
$$

Similarly we can define a Schwarz algorithm for the time-harmonic Maxwell equations in a given domain $\Omega=\mathbb{R}^{3}$

$$
-i \omega \varepsilon \mathbf{E}+\nabla \times \mathbf{H}=\mathbf{J}, \quad i \omega \mu \mathbf{H}+\nabla \times \mathbf{E}=\mathbf{0},
$$

with the Silver-Müller radiation condition [25, 22]

$$
\lim _{r \rightarrow \infty} r\left(\mathbf{H} \times e_{\mathbf{r}}+\frac{1}{Z_{j}} \mathbf{E}\right)=0,
$$

where $r:=|\mathbf{x}|$ and $e_{\mathbf{r}}=\mathbf{x} / r$ for any vector $\mathbf{x} \in \mathbb{R}^{3}$.

We also consider the heterogeneous case where the domain $\Omega$ consists of two non-overlapping subdomains $\Omega_{1}:=\mathbb{R}^{-} \times \mathbb{R}^{2}$ and $\Omega_{2}:=\mathbb{R}^{+} \times \mathbb{R}^{2}$ with interface $\Gamma$, with piece-wise constant parameters $\varepsilon_{j}$ and $\mu_{j}$ in $\Omega_{j}, j=1,2$. A general Schwarz algorithm for this configuration is

$$
\begin{array}{ccc}
-i \omega \varepsilon_{1} \mathbf{E}^{1, n}+\nabla \times \mathbf{H}^{1, n}=\mathbf{J}, & i \omega \mu_{1} \mathbf{H}^{1, n}+\nabla \times \mathbf{E}^{1, n}=\mathbf{0} & \text { in } \Omega_{1}, \\
\left(\mathcal{B}_{\mathbf{n}_{1}}+\mathcal{S}_{1} \mathcal{B}_{\mathbf{n}_{2}}\right)\left(\mathbf{E}^{1, n}, \mathbf{H}^{1, n}\right)=\left(\mathcal{B}_{\mathbf{n}_{1}}+\mathcal{S}_{1} \mathcal{B}_{\mathbf{n}_{2}}\right)\left(\mathbf{E}^{2, n-1}, \mathbf{H}^{2, n-1}\right) & \text { on } \Gamma, \\
-i \omega \varepsilon_{2} \mathbf{E}^{2, n}+\nabla \times \mathbf{H}^{2, n}=\mathbf{J}, & i \omega \mu_{2} \mathbf{H}^{2, n}+\nabla \times \mathbf{E}^{2, n}=\mathbf{0} & \text { in } \Omega_{2}, \\
\left(\mathcal{B}_{\mathbf{n}_{2}}+\mathcal{S}_{2} \mathcal{B}_{\mathbf{n}_{1}}\right)\left(\mathbf{E}^{2, n}, \mathbf{H}^{2, n}\right)=\left(\mathcal{B}_{\mathbf{n}_{2}}+\mathcal{S}_{2} \mathcal{B}_{\mathbf{n}_{1}}\right)\left(\mathbf{E}^{1, n-1}, \mathbf{H}^{1, n-1}\right) & \text { on } \Gamma,
\end{array}
$$

where $\mathcal{S}_{j}, j=1,2$ are tangential, possibly pseudo-differential operators, and

$$
\mathcal{B}_{\mathbf{n}_{j}}\left(\mathbf{E}^{j, n}, \mathbf{H}^{j, n}\right)=\frac{\mathbf{E}^{j, n}}{Z_{j}} \times \mathbf{n}_{j}+\mathbf{n}_{j} \times\left(\mathbf{H}^{j, n} \times \mathbf{n}_{j}\right)
$$

are the characteristic conditions, with $Z_{j}=\sqrt{\mu_{j} / \epsilon_{j}}, j=1,2$. Different choices of $\mathcal{S}_{j}, j=1,2$ lead to different Schwarz methods, see [11].

Remark 1. A direct computation shows that algorithms (3) and (8) have the same convergence factor, when setting $\rho_{j}:=\mu_{j}$ and $c_{j}:=\frac{1}{\sqrt{\varepsilon_{j} \mu_{j}}}$ for $j=1,2$. Hence we can use all the results presented in [9] for Maxwell's equations for the case of the Helmholtz equation (3). We thus focus in the remainder on the Helmholtz case, but keep in mind that all results we will obtain hold mutatis mutandis also for the Maxwell case.

Using Remark 1, we obtain from [9] and [10]

Corollary 1. The solution of (5) for $c_{1} \neq c_{2}$ is asymptotically

$$
\rho_{\text {opt }}^{*}= \begin{cases}1-\mathcal{O}\left(h^{1 / 4}\right) & \text { if } \rho_{1}=\rho_{2}, \\ \sqrt{\frac{\rho_{\min }}{\rho_{\max }}}+\mathcal{O}\left(h^{1 / 2}\right) & \text { if } \frac{1}{\sqrt{2}} \leq \frac{\rho_{1}}{\rho_{2}} \leq \sqrt{2}, \\ \sqrt[4]{\frac{1}{2}}+\mathcal{O}\left(h^{1 / 2}\right) & \text { if } \frac{\rho_{1}}{\rho_{2}}<\frac{1}{\sqrt{2}} \text { or } \frac{\rho_{1}}{\rho_{2}}>\sqrt{2} .\end{cases}
$$


If $\rho_{1} \neq \rho_{2}$ and $c_{1}=c_{2}$, we obtain after excluding the resonance frequency [11]

$$
\rho_{\text {opt }}^{*}=\sqrt{\frac{\rho_{\min }}{\rho_{\max }}}+\mathcal{O}\left(h^{1 / 2}\right),
$$

with $\rho_{\min }=\min \left\{\rho_{1}, \rho_{2}\right\}$ and $\rho_{\max }=\max \left\{\rho_{1}, \rho_{2}\right\}$.

The detailed proof of Corollary 1 and the values of $P_{j}$ can be found in [27]. We see from Corollary 1 that in most of the cases the optimized convergence factor $\rho_{\mathrm{opt}}^{*}$ has an asymptotic behavior independent of the mesh size $h$.

\section{Scaling Results when Controlling the Pollution Effect}

The core of our study is the asymptotic analysis of algorithms (3) and (8) when the mesh size $h$ is related to the wave number $\omega$ to control the pollution effect [2]. We will focus on the first case of Corollary 1 , because this is the only case where the convergence can deteriorate in the mesh size $h$; see the first line in (9). We will consider three particular relationships between $\omega$ and $h$ : $\omega h=C_{\omega}, C_{\omega}$ a constant, where the pollution effect is not controlled, $\omega^{2} h=$ $C_{\omega}$ where the pollution effect is provably controlled, and finally $\omega^{3 / 2} h=C_{\omega}$ which is widely believed to suffice to control the pollution effect.

Theorem 1. Let $\rho_{1}=\rho_{2}, c_{1} \neq c_{2}$ and $\omega h=C_{\omega}$. If $\left|\rho_{\text {opt }}\right|$ defined in (4) is maximal for the frequencies $k=\omega_{1}, k=\omega_{2}$ and $k=k_{\max }$, and $s_{j}=(1+i) P_{j}$, then the solution of the min-max problem (5) is

$$
P_{1}^{*}=\frac{\bar{p}_{1}}{h}, \quad P_{2}^{*}=\frac{\bar{p}_{2}}{h}, \quad \rho_{o p t}^{*}=\left(\frac{\bar{p}_{1}^{2}\left(2 \bar{p}_{2}^{2}-2 \bar{p}_{2} c_{r}+c_{r}^{2}\right)}{\bar{p}_{2}^{2}\left(2 \bar{p}_{1}^{2}+2 \bar{p}_{1} c_{r}+c_{r}^{2}\right)}\right)^{\frac{1}{4}},
$$

where $\left\{\bar{p}_{1}, \bar{p}_{2}\right\}$ is solution of the system of equations

$$
\begin{aligned}
& \frac{p_{1}^{2}\left(2 p_{2}^{2}-2 p_{2} c_{r}+c_{r}^{2}\right)}{p_{2}^{2}\left(2 p_{1}^{2}+2 p_{1} c_{r}+c_{r}^{2}\right)}=\frac{\rho^{2} p_{2}^{2}\left(2 p_{1}^{2}-2 p_{1} c_{r}+c_{r}^{2}\right)}{p_{1}^{2}\left(2 p_{2}^{2}+2 p_{2} c_{r}+c_{r}^{2}\right)},
\end{aligned}
$$

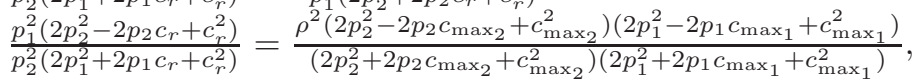

$c_{r}:=r h:=\sqrt{\left|\omega_{1}^{2}-\omega_{2}^{2}\right|} h, c_{\max _{1}}:=\sqrt{c_{\max }^{2}-C_{\omega}^{2} / c_{1}^{2}}, c_{\max _{2}}:=\sqrt{c_{\max }^{2}-C_{\omega}^{2} / c_{2}^{2}}$.

Proof. Evaluating $\left|\rho_{\text {opt }}\right|^{4}$ from (4) at $s_{j}:=\frac{p_{j}}{h}(1+i)$ for $k=\omega_{1}, k=\omega_{2}$ and $k=k_{\max }$ yields

$$
\begin{aligned}
& R_{1}=\frac{\left(h^{2} r^{2}-2 p_{2} h r+2 p_{2}^{2}\right) p_{1}^{2}}{p_{2}^{2}\left(h^{2} r^{2}+2 p_{1} h r+2 p_{1}^{2}\right.}, \quad R_{2}=\frac{\rho^{2} p_{2}^{2}\left(h^{2} r^{2}-2 p_{1} h r+2 p_{1}^{2}\right)}{\left(2 p_{2}^{2}+2 p_{2} h r+h^{2} r^{2}\right) p_{1}^{2}}, \\
& R_{3}=\frac{\left(h^{2}\left(\frac{c_{\max }^{2}}{h^{2}}-\frac{C_{\omega}^{2}}{c_{2}^{2} h^{2}}\right)-2 p_{2} h \sqrt{\frac{c_{\max }^{2}}{h^{2}}-\frac{C_{\omega}^{2}}{c_{2}^{2} h^{2}}}+2 p_{2}^{2}\right)}{\left(h^{2}\left(\frac{c_{\max }^{2}}{h^{2}}-\frac{C_{\omega}^{2}}{c_{2}^{2} h^{2}}\right)-2 p_{1} h \sqrt{\frac{c_{\max }^{2}}{h^{2}}-\frac{C_{\omega}^{2}}{c_{2}^{2} h^{2}}}+2 p_{1}^{2}\right)} \frac{\left(h^{2}\left(\frac{c_{\max }^{2}}{h^{2}}-\frac{C_{\omega}^{2}}{c_{1}^{2} h^{2}}\right)-2 p_{1} h \sqrt{\frac{c_{\max }^{2}}{h^{2}}-\frac{C_{\omega}^{2}}{c_{1}^{2} h^{2}}}+2 p_{1}^{2}\right)}{\left(h^{2}\left(\frac{c_{\max }^{2}}{h^{2}}-\frac{C_{\omega}^{2}}{c_{1}^{2} h^{2}}\right)-2 p_{2} h \sqrt{\frac{c_{\max }^{2}}{h^{2}}-\frac{C_{\omega}^{2}}{c_{1}^{2} h^{2}}}+2 p_{2}^{2}\right)} .
\end{aligned}
$$


Replacing $r h$ by $c_{r}, c_{\max _{1}}=\sqrt{c_{\max }^{2}-C_{\omega}^{2} / c_{1}^{2}}$ and $c_{\max _{2}}=\sqrt{c_{\max }^{2}-C_{\omega}^{2} / c_{2}^{2}}$, the expressions can be simplified to

$$
\begin{aligned}
R_{1} & =\frac{p_{1}^{2}\left(2 p_{2}^{2}-2 p_{2} c_{r}+c_{r}^{2}\right)}{p_{2}^{2}\left(2 p_{1}^{2}+2 p_{1} c_{r}+c_{r}^{2}\right)}, \quad R_{2}=\frac{\rho^{2} p_{2}^{2}\left(2 p_{1}^{2}-2 p_{1} c_{r}+c_{r}^{2}\right)}{p_{1}^{2}\left(2 p_{2}^{2}+2 p_{2} c_{r}+c_{r}^{2}\right)} \\
R_{3} & =\frac{\left(2 p_{2}^{2}-2 p_{2} c_{\max _{2}}+c_{\max _{2}}^{2}\right)\left(2 p_{1}^{2}-2 p_{1} c_{\max _{1}}+c_{\max _{1}}^{2}\right)}{\left(2 p_{2}^{2}+2 p_{2} c_{\max _{2}}+c_{\max _{2}}^{2}\right)\left(2 p_{1}^{2}+2 p_{1} c_{\max _{1}}+c_{\max _{1}}^{2}\right)}
\end{aligned}
$$

Equioscillation between $R_{1}, R_{2}$ and $R_{3}$ then gives the result [27].

Remark 2. Note that Theorem 1 gives a closed form solution of the min-max problem (5), not just an asymptotic one.

For the special case of equal transmission conditions, we have

Corollary 2. Under the same assumptions as in Theorem 1, if $s_{j}=(1+i) P_{j}$ with $P_{1}=P_{2}$, then the solution of the min-max problem (5) is given by

$$
P_{1}^{*}=P_{2}^{*}=\frac{\bar{p}}{h}, \quad \rho_{o p t}^{*}=\left(\frac{\left(2 \bar{p}^{2}-2 \bar{p} c_{r}+c_{r}^{2}\right)}{\left(2 \bar{p}^{2}+2 \bar{p} c_{r}+c_{r}^{2}\right)}\right)^{\frac{1}{4}},
$$

with $\bar{p}$ the solution of the equation

$$
\frac{\left(2 p^{2}-2 p c_{r}+c_{r}^{2}\right)}{\left(2 p^{2}+2 p c_{r}+c_{r}^{2}\right)}=\frac{\left(2 p^{2}-2 p c_{\max _{2}}+c_{\max _{2}}^{2}\right)\left(2 p^{2}-2 p c_{\max _{1}}+c_{\max _{1}}^{2}\right)}{\left(2 p^{2}+2 p c_{\max _{2}}+c_{\max _{2}}^{2}\right)\left(2 p^{2}+2 p c_{\max _{1}}+c_{\max _{1}}^{2}\right)} .
$$

Proof. The proof follows along the same lines as the proof of Theorem 1.

Theorem 2. Let $\rho_{1}=\rho_{2}, c_{1} \neq c_{2}$ and $\omega^{2} h=C_{\omega}$. If $\left|\rho_{\text {opt }}\right|$ defined in (4) is maximal for the frequencies $k=\omega_{1}, k=\omega_{2}, k=k_{m}:=\frac{c_{m}}{h^{3 / 4}}$ and $k=k_{\max }$, and $s_{j}=(1+i) P_{j}, P_{1}=\frac{p_{1}}{h}$ and $P_{2}=\frac{p_{2}}{\sqrt{h}}$, then the asymptotic solution of the min-max problem (5) for $h$ small is given by

$$
P_{1}^{*}=\frac{c_{\max }^{3 / 4} c_{r}^{1 / 4}}{2^{1 / 4} h^{7 / 8}}, \quad P_{2}^{*}=\frac{1}{2} \frac{c_{\max }^{1 / 4} c_{r}^{3 / 4}}{2^{3 / 4} h^{5 / 8}}, \quad \rho_{o p t}^{*}=1-\frac{r^{1 / 4}}{2^{1 / 4} c_{\max }^{1 / 4}} h^{1 / 8}+\mathcal{O}\left(h^{1 / 4}\right) .
$$

Interchanging the role of $P_{1}$ and $P_{2}$ leads to the same result.

Proof. The proof is based again on equioscillation.

Theorem 3. Let $\rho_{1}=\rho_{2}, c_{1} \neq c_{2}$ and $\omega^{3 / 2} h=C_{\omega}$. If the frequencies $k=\omega_{1}$, $k=\omega_{2}, k=k_{m}:=\frac{c_{m}}{h^{5 / 6}}$ and $k=k_{\max }$ are the local maxima of the convergence factor $\rho_{\text {opt }}$ from (4), and if $s_{1}=(1+i) P_{1}, s_{2}=(1+i) P_{2}$, with $P_{1}=\frac{p_{1}}{h^{11 / 12}}$ and $P_{2}=\frac{p_{2}}{h^{3 / 4}}$, then the asymptotic solution of the min-max problem (5) for $h$ small is given by

$$
P_{1}^{*}=\frac{c_{\max }^{3 / 4} c_{r}^{1 / 4}}{2^{1 / 4} h^{11 / 12}}, \quad P_{2}^{*}=\frac{1}{2} \frac{c_{\max }^{1 / 4} c_{r}^{3 / 4}}{2^{3 / 4} h^{3 / 4}}, \quad \rho_{\text {opt }}^{*}=1-\frac{r^{1 / 4}}{2^{1 / 4} c_{\max }^{1 / 4}} h^{1 / 12}+\mathcal{O}\left(h^{1 / 6}\right) .
$$




\begin{tabular}{|c|c|c|c|c|}
\hline & $\omega=C_{\omega}$ & $\omega^{2} h=C_{\omega}$ & $\omega^{3 / 2} h=C_{\omega}$ & $\omega h=C_{\omega}$ \\
\hline$\rho_{1}=\rho_{2}, c_{1} \neq c_{2}$ & $\begin{array}{c}1-\mathcal{O}\left(h^{1 / 4}\right) \\
(\text { Corollary 1) }\end{array}$ & $\begin{array}{c}1-\mathcal{O}\left(h^{1 / 8}\right) \\
(\text { Theorem 2) }\end{array}$ & $\begin{array}{c}1-\mathcal{O}\left(h^{1 / 12}\right) \\
(\text { Theorem 3) }\end{array}$ & $\begin{array}{c}<1 \\
(\text { Theorem 1) }\end{array}$ \\
\hline$\rho_{1} \neq \rho_{2}, c_{1} \neq c_{2}$ & $\begin{array}{c}\max \left\{\sqrt[4]{\frac{1}{2}}, \sqrt{\frac{\rho_{\min }}{\rho_{\max }}}\right\} \\
(\text { Corollary 1) }\end{array}$ & $\begin{array}{c}\max \left\{\sqrt[4]{\frac{1}{2}}, \sqrt{\frac{\rho_{\min }}{\rho_{\max }}}\right\} \\
(\text { Remark 3) }\end{array}$ & $\begin{array}{c}\max \left\{\sqrt[4]{\frac{1}{2}}, \sqrt{\frac{\rho_{\min }}{\rho_{\max }}}\right\} \\
(\text { Remark 3) }\end{array}$ & $\begin{array}{c}<1 \\
(\text { Remark 3) }\end{array}$ \\
\hline$\rho_{1} \neq \rho_{2}, c_{1}=c_{2}$ & $\begin{array}{c}\sqrt{\frac{\rho_{\min }}{\rho_{\max }}} \\
(\text { Corollary 1) }\end{array}$ & $\begin{array}{c}\sqrt{\frac{\rho_{\min }}{\rho_{\max }}} \\
(\text { Remark 3) }\end{array}$ & $\begin{array}{c}\sqrt{\frac{\rho_{\min }}{\rho_{\max }}} \\
(\text { Remark 3) }\end{array}$ & $\begin{array}{c}<1 \\
(\text { Remark 3) }\end{array}$ \\
\hline
\end{tabular}

Table 1 Comparison of the convergence factors with different relationships between $\omega$ and $h$.

Interchanging the role of $P_{1}$ and $P_{2}$ leads to the same result.

Proof. The proof is similar to the proof of Theorem 2.

One can justify the choice of the frequencies $k=\omega_{1}, k=\omega_{2}, k=k_{m}$ and $k=k_{\max }$ as the correct candidates for the $\left|\rho_{\text {opt }}\right|$ using asymptotic analysis, but this exceeds the space available, see [27] for more details.

Remark 3. One can obtain similar results also for the cases $\rho_{1} \neq \rho_{2}$ but this will only reduce the order of the second asymptotic term, as in Theorems 2 and 3. For the relationship $\omega h=C_{\omega}$ one can also obtain a similar result to Theorem 1.

We give a summary of all these results in Table 1.

\section{Conclusions}

We studied the performance of optimized Schwarz methods for Helmholtz and Maxwell's equations for heterogeneous media, where aligning interfaces with heteronegeities can lead to methods that converge faster than without heterogeneities. This is in contrast to recent approaches like GenEO [26] that lead to robust methods for heterogeneous media without alignment of interfaces, but they can not benefit from the heterogeneity. Using Fourier analysis, we showed that the convergence factor of the optimized Schwarz methods for the Helmholtz equation and the Maxwell's equations are the same, and it suffices therefore to study the algorithms only for the Helmholtz equation. We then studied in detail the performance for three different choices of the relationship between the wave number and the mesh size to control the pollution effect, and showed that increasing the resolution improves the performance of the optimized Schwarz methods. It was not possible to show all the proofs in detail in this short manuscript, but more information can be found in the $\mathrm{PhD}$ thesis [27]. 


\section{References}

[1] A. Alonso-Rodriguez and L. Gerardo-Giorda. New nonoverlapping domain decomposition methods for the harmonic Maxwell system. SIAM J. Sci. Comput., 28(1):102-122, 2006.

[2] I. M. Babuska and S. A. Sauter. Is the pollution effect of the FEM avoidable for the Helmholtz equation considering high wave numbers? SIAM J. Numer. Anal., 34(6):2392-2423, 1997.

[3] Y. Boubendir, X. Antoine, and C. Geuzaine. A quasi-optimal nonoverlapping domain decomposition algorithm for the Helmholtz equation. J. Comput. Phys., 231(2):262-280, 2012.

[4] Z. Chen and X. Xiang. A source transfer domain decomposition method for Helmholtz equations in unbounded domain. SIAM J. Numer. Anal., 51(4):2331-2356, 2013.

[5] P. Chevalier and F. Nataf. An OO2 (Optimized Order 2) method for the Helmholtz and Maxwell equations. In 10th International Conference on Domain Decomposition Methods in Science and in Engineering, pages 400-407. AMS, 1997.

[6] B. Després. Décomposition de domaine et problème de Helmholtz. C.R. Acad. Sci. Paris, 1(6):313-316, 1990.

[7] B. Després, P. Joly, and J. E. Roberts. A domain decomposition method for the harmonic Maxwell equations. In Iterative methods in linear algebra, pages 475-484, Amsterdam, 1992. North-Holland.

[8] V. Dolean, M. El Bouajaji, M. J. Gander, S. Lanteri, and R. Perrussel. Domain decomposition methods for electromagnetic wave propagation problems in heterogeneous media and complex domains. In Domain decomposition methods in science and engineering XIX, volume 78 of Lect. Notes Comput. Sci. Eng., pages 15-26. Springer, Heidelberg, 2011.

[9] V. Dolean, M. J. Gander, and E. Veneros. Optimized Schwarz methods for Maxwell equations with discontinuous coefficients. In Domain Decomposition Methods in Science and Engineering XXI, Lecture Notes in Computational Science and Engineering, pages 517-526. Springer, Heidelberg, 2014.

[10] V. Dolean, M. J. Gander, and E. Veneros. Schwarz methods for second order Maxwell equations in 3d with coefficient jumps. In Domain Decomposition Methods in Science and Engineering XXII, Lecture Notes in Computational Science and Engineering. Springer, Heidelberg, 2015.

[11] V. Dolean, L. Gerardo-Giorda, and M.J. Gander. Optimized Schwarz methods for Maxwell equations. SIAM J. Scient. Comp., 31(3):21932213, 2009.

[12] V. Dolean, S. Lanteri, and R. Perrussel. A domain decomposition method for solving the three-dimensional time-harmonic Maxwell equations discretized by discontinuous Galerkin methods. J. Comput. Phys., $227(3): 2044-2072,2008$. 
[13] V. Dolean, S. Lanteri, and R. Perrussel. Optimized Schwarz algorithms for solving time-harmonic Maxwell's equations discretized by a discontinuous Galerkin method. IEEE. Trans. Magn., 44(6):954-957, 2008.

[14] O. Dubois. Optimized Schwarz Methods for the Advection-Diffusion Equation and for Problems with Discontinuous Coefficients. PhD thesis, McGill University, June 2007.

[15] O. Dubois and M. J. Gander. Optimized Schwarz methods for a diffusion problem with discontinuous coefficient. Numer. Algorithms, 69(1):109144, 2015.

[16] M. El Bouajaji, V. Dolean, M. J. Gander, and S. Lanteri. Optimized Schwarz methods for the time-harmonic Maxwell equations with damping. SIAM J. Sci. Comput., 34(4):A2048-A2071, 2012.

[17] Björn Engquist and Lexing Ying. Sweeping preconditioner for the Helmholtz equation: hierarchical matrix representation. Comm. Pure Appl. Math., 64(5):697-735, 2011.

[18] O. G. Ernst and M. J. Gander. Why it is difficult to solve Helmholtz problems with classical iterative methods. In Numerical analysis of multiscale problems, volume 83 of Lect. Notes Comput. Sci. Eng., pages 325-363. Springer, Heidelberg, 2012.

[19] M. J. Gander. Optimized Schwarz methods for Helmholtz problems. In Thirteenth international conference on domain decomposition, pages 245-252. CIMNE, Barcelona, Spain, 2001.

[20] M. J. Gander, L. Halpern, and F. Magoulès. An optimized Schwarz method with two-sided Robin transmission conditions for the Helmholtz equation. Int. J. for Num. Meth. in Fluids, 55(2):163-175, 2007.

[21] M.J. Gander, F. Magoulès, and F. Nataf. Optimized Schwarz methods without overlap for the Helmholtz equation. SIAM J. Sci. Comput., 24(1):38-60, 2002.

[22] C. Müller. Grundprobleme der mathematischen Theorie elektromagnetischer Schwingungen. Springer, 1957.

[23] Z. Peng and J. F. Lee. Non-conformal domain decomposition method with second-order transmission conditions for time-harmonic electromagnetics. J. Comput. Physics, 229(16):5615-5629, 2010.

[24] Z. Peng, V. Rawat, and J. F. Lee. One way domain decomposition method with second order transmission conditions for solving electromagnetic wave problems. J. Comput. Physics, 229(4):1181-1197, 2010.

[25] S. Silver. Microwave antenna theory and design. Number 19. Iet, 1949.

[26] N. Spillane, V. Dolean, P. Hauret, F. Nataf, C. Pechstein, and R. Scheichl. Abstract robust coarse spaces for systems of PDEs via generalized eigenproblems in the overlaps. Numer. Mathematik, 126(4):741-770, 2014.

[27] E. Veneros. Méthodes des décomposition de domaines pour des problèmes de propagation d'ondes heterogènes. PhD thesis, University of Geneva, 2015. 